\title{
Dr. Kathryn Kell erhält ersten „IDM Oral Health Progress Award”
}

Die Internationale Dentalherstellervereinigung IDM hat Dr. Kathryn Kell aus den USA den ersten globalen MundgesundheitsFörderungspreis „IDM Global Oral Health Progress Award“ verliehen. Der Preis würdigt Kells Beitrag zur Förderung der Mundgesundheit, insbesondere ihre führende Rolle bei den Task-Force-Initiativen der Kongresse des FDI World Dental Federation.

Die neu ins Leben gerufene Auszeichnung, die jährlich verliehen wird, umfasst eine Spende, die im Namen der Preisträgerin an ein Charity-Projekt geht, das sie selbst aussucht. „Dr.
Kathryn Kell hat ihre Managementerfahrung großzügig in die Task Force eingebracht und dadurch die Zusammenarbeit zwischen der FDI und der IDM auf eine neue Ebene gestellt. Sie hat die Zukunft des FDI-Kongresses auf allen Ebenen neu gestaltet“, sagte IDM-Präsident Christian Brutzer.

Dr. Kell, die derzeit als President-Elect der FDI verantwortlich zeichnet, war mehr als 40 Jahre lang als Zahnärztin tätig und hatte in den vergangenen 17 Jahren mehrere führende Funktionen in der FDI inne. Die FDI World Dental Federation ist die Stimme von über einer Million Zahnärzten weltweit.

\section{Erster Preis für Hamburger Team}

\section{CharityAward 2015 für "Praxis ohne Grenzen“}

Mit einem großen Team aus Ärzten, Krankenschwestern, Medizinisch-technischen Assistenten sowie Dolmetschern behandelt Professor Dr. Peter C. Ostendorf in der Hamburger „Praxis ohne Grenzen“ Menschen, die aus verschiedenen Gründen nicht versichert sind. Und das macht er völlig kostenlos. Dafür wurde ihm der erste Preis des CharityAwards 2015 verliehen.

Ostendorf und sein Team helfen Patienten, die beispielsweise nicht krankenversichert sind, weil sie als Selbstständige in die Insolvenz geraten sind und sich die Versicherung nicht mehr leisten können, oder Obdachlose, Asylsuchende, Flüchtlinge ohne Aufenthaltsstatus und EU-Bürger aus Osteuropa, die noch keine sozialversicherungspflichtige Arbeit in Deutschland gefunden haben.
Ostendorf, ehemaliger Chefarzt des Zentrums für Innere Medizin im Hamburger Marienkrankenhaus, startete die Initiative im Frühjahr 2014. Im ersten Jahr kamen insgesamt 1.280 Patienten in die Sprechstunde der „Praxis ohne Grenzen“. Der CharityAward ist verbunden mit einem Preisgeld von 20.000 Euro.

Am 20. Oktober 2016 wird Springer Medizin den nächsten CharityAward verleihen. Dotiert ist der Preis mit einem Preisgeld von insgesamt 60.000 Euro und einem Medienpaket, aufgeteilt auf drei Gewinner. Bewerbungen an Springer Medizin GmbH, Sabine Springer, Postfach 21 31, 63243 Neu-Isenburg, E-Mail: charity@springer.com. Infos unter www.springermedizin.de/charity

\section{Auf dem neuesten Stand mit wissen kompakt}

Schmerzen im Zahn-, Mund- und Kieferbereich gehören zum wichtigsten Grund für einen Zahnarztbesuch. Fallen Diagnostik und Therapie bei akuten Schmerzen meist adäquat und zielgerichtet aus, bestehen bei chronischen Schmerzen jedoch oft Unsicherheiten. Häufig werden Patienten mit chronischen Schmerzen nicht rechtzeitig erkannt, therapiert oder überwiesen. Teilweise kommt es auch zu Fehldiagnosen. Aus diesem Grund widmet sich die Mai-Ausgabe von wissen kompakt dem komplexen Thema Schmerz.

\section{"Trigeminusneuropathie"}

von Prof. Dr. Dr. M. Daubländer, Dr. C. Welte-Jzyk

\author{
"Multimodale Therapie bei Patienten mit \\ Gesichtsschmerzen" \\ von PD Dr. Dr. D. A. Ettlin, lic. phil. B. Steiger, \\ Dr. med. et med. dent. N. Lukic
}

\section{„Therapieoptionen bei Kiefergelenkschmerzen" von Dr. H. Kares}

Die Teilnahme an den Continuing-Medical-Education(CME)Fortbildungen erfolgt online auf www.springerzahnmedizin.de und ist für die Mitglieder des Freien Verbands Deutscher Zahnärzte (FVDZ) sowie für Abonnenten von wissen kompakt kostenlos. Beides trifft nicht auf Sie zu? Die entsprechenden Anträge finden Sie in dieser Ausgabe auf Seite 57/58.

wissen kompakt - gebündelte zertifizierte zahnärztliche Fortbildung 